\title{
Phenomenology of Social Cognition
}

\author{
Shannon Spaulding
}

(Forthcoming in Erkenntnis)

\begin{abstract}
:
Can phenomenological evidence play a decisive role in accepting or rejecting social cognition theories? Is it the case that a theory of social cognition ought to explain and be empirically supported by our phenomenological experience? There is serious disagreement about the answers to these questions. This paper aims to determine the methodological role of phenomenology in social cognition debates. The following three features are characteristic of evidence capable of playing a substantial methodological role: novelty, reliability, and relevance. I argue that phenomenological evidence lacks all three criteria and, consequently, should not play a substantial role in debates about social cognition.
\end{abstract}

\section{Introduction}

Social cognition is our ability to understand and interact with other agents. It is a

ubiquitous feature of our lives. Talking to a colleague, driving on a busy freeway, teaching, and taking care of one's children are just a few examples of behaviors that involve social cognition. Each of these events involves interpreting others' behavior, anticipating what they will do next, and facilitating interaction with other agents.

Phenomenology is the study of conscious experiences from the first-person perspective. ${ }^{1}$ Some theorists regard phenomenology as crucially important to the study of

1 The focus of this paper is what I call phenomenological reports. These are first-person reports on one's conscious experiences. Examples include reporting that I am experiencing a painful sensation, seeing a yellow patch, feeling angry, and thinking about what I had for breakfast. In each case, I am reporting what I am (or I take myself to be) consciously experiencing. Though this terminology is standard in the social cognition debates (Gallagher, 2012; Herschbach, 2008a; Spaulding, 2010; Zahavi, 2011) and more generally 
social cognition (De Jaegher \& Di Paolo, 2007; Fuchs, 2012; Gallagher, 2012; Ratcliffe, 2006;

Zahavi, 2011). According to this view, phenomenological evidence about our social interactions can and should be marshaled as evidence for or against theories of social cognition. To this end, these theorists contend that the phenomenological evidence supports their theories of social cognition, variously called Interaction Theory, Embodied Cognition, and Enactive Cognition. Moreover, they argue that the phenomenological evidence disconfirms alternative accounts, e.g., the Theory Theory, the Simulation Theory, and hybrid versions of the two accounts. These theories, it is argued, predict conscious experiences that phenomenology shows we do not have, and so we should reject these theories. $^{2}$

In contrast, other theorists argue that phenomenological evidence is not so important and perhaps even is irrelevant to the debates about social cognition (Carruthers, 2009; Currie, 2008; Herschbach, 2008b; Jacob, 2011; Spaulding, 2010). Proponents of this view hold that the relevant debates in the social cognition literature concern the cognitive architecture responsible for our ability to understand and interact with other agents, and phenomenology tells us very little about cognitive architecture.

There is one point of agreement in this dispute between phenomenologists and nonphenomenologists. All parties to this debate accept - or, in any case, ought to accept - that in empirically oriented analytic philosophy of mind (Dennett, 2003; Noë, 2007), continental philosophers in the phenomenological tradition would regard "phenomenological reports" as an oxymoron because to verbally express one's phenomenology is to defeat the purpose of phenomenology. To verbalize one's experiences is to impose a linguistic, cultural framework on the experience, which thwarts the goal of studying the experience itself. For the sake of continuity with the ongoing debate, I will continue to use the terminology "phenomenological reports."

2 The case for interactive, embodied, and enactive accounts of social cognition does not rest wholly on this phenomenological argument. Proponents of these views also claim support from developmental psychology and the alleged failure of the main alternatives, the Theory 
phenomenology at least weakly constrains the study of social cognition. Specifically, a theory of social cognition ought to be compatible with our phenomenological experience of social interactions. In other words, it would count against a theory if it entailed a phenomenology radically different from what we experience. ${ }^{3}$ This is a very weak constraint. A theory could satisfy this constraint simply by having no implications for our phenomenological experience of social interactions. If the claim at issue is that phenomenology only weakly constrains the study of social cognition, phenomenology would not have any special methodological role in debates about social cognition, and there would be no serious disagreement between the two views described above.

Highlighting this point of agreement brings into focus the true disagreement between phenomenologists and non-phenomenologists. This disagreement concerns whether phenomenology strongly constrains the study of social cognition. To settle this disagreement we have to determine whether phenomenology plays a more substantial methodological role. For instance, can phenomenological evidence play a decisive role in accepting or rejecting social cognition theories? Is it the case that a theory of social cognition ought to explain and be empirically supported by our phenomenological experience? Some theorists employ phenomenological evidence in just this way (Gallagher, 2012; Zahavi, 2011). Notice that this is a much stronger constraint that many theories of

${ }^{3}$ Another potential point of agreement is that a completed account of social cognition will explain how the sub-personal mechanisms posited enable the conscious social experiences we have. However, no theorist purports to have a completed account of social cognition yet, and phenomenologists and non-phenomenologists disagree about the methodological role of phenomenology in the current theoretical context. They even disagree about whether phenomenology is a reliable guide to the nature of social cognition, e.g., whether social cognition is perceptually based or inferentially based (Spaulding, forthcoming). Thus, this point of agreement about a completed account of social cognition has little bearing on the current debate. 
social cognition would not satisfy, e.g., any theory that has no implications about our phenomenology.

Whether this stronger constraint on theories of social cognition is legitimate depends on the nature of the phenomenological evidence. The following three features are characteristic of empirical evidence capable of playing a substantial methodological role: novelty, reliability, and relevance. Evidence is novel when it provides information beyond what is widely acknowledged and presupposed by the theories debated. It is reliable insofar as it is consistent, accurate, and unbiased. Evidence is relevant to the extent that it can confirm or disconfirm some of the hypotheses debated. We need not quibble over how to assign weights to each characteristic or whether all three characteristics are, strictly speaking, necessary. It is enough to note that, in general, empirical evidence capable of playing a substantial methodological role has these three features. Hence, for proponents of phenomenology the task is to establish that phenomenological evidence has at least some of these characteristics.

In this paper, I argue that phenomenological evidence has none of these three characteristics. Consequently, phenomenology should not play a substantial role in debates about social cognition. If successful, this argument will show that the phenomenological arguments employed in the social cognition debate are wrongheaded and ought to be disregarded.

\section{Phenomenology of Social Cognition: Summarizing the Debate}


Traditionally, philosophers and psychologists have assumed that in order to understand and successfully interact with other people we must understand how mental states, such as beliefs, desires, and intentions, inform behavior and how behavior affects mental states. The mainstream view is that adult human social cognition fundamentally involves mindreading, i.e., attributing mental states to others in order to understand, anticipate, and influence their behavior. Two competing accounts have dominated the mindreading literature: the Theory Theory (TT) and the Simulation Theory (ST).

The TT holds that we explain and predict behavior by employing a tacit folk psychological theory about how mental states inform behavior. With our folk psychological theory, we infer from a target's behavior what his or her mental states probably are. From these inferences, plus the psychological principles in the theory connecting mental states to behavior, we predict the target's behavior.

The ST, in contrast, holds that we explain and predict a target's behavior by using our own minds as a simulation of the other person's mind. To explain a target's behavior, we put ourselves in another's shoes, so to speak, and imagine what our mental states would be and how we would behave if we were that agent in that particular situation. To predict a target's behavior, we take the attributed mental states as input and simulate the target's decision about what to do next.

The TT and the ST disagree about how mindreading works. The TT contends that it is an information-rich theoretical process, whereas the ST maintains that it is an information-poor simulational process. Though TT and ST proponents disagree about how mindreading operates, they agree that mindreading is pervasive and the primary way we understand others. They also agree that much of mindreading occurs subconsciously. Both 
theorizing and simulating may be conscious, deliberative processes or spontaneous, subconscious processes.

Phenomenologists argue that both the TT and the ST are misguided accounts of social cognition. They contend that there is good phenomenological evidence against the claim that mindreading is pervasive and our primary mode of understanding others (De Jaegher \& Di Paolo, 2007; Fuchs, 2012; Gallagher, 2012; Hutto, 2008; Ratcliffe, 2006; Zahavi, 2011). Both TT and ST hold that we resort to third-person based explanation and prediction in our ordinary social interactions. According to critics of mindreading, this implies that we adopt a spectator's perspective on our interactions. However, phenomenology tells us that we rarely adopt a spectator's perspective. Relying on introspection-based reports on our experience of social interactions, phenomenologists argue that our ordinary interactions do not involve explanation and prediction and they are not third-person based attempts to theorize about or simulate mental states. Thus, phenomenology tells us that mindreading is rare and hence not our primary mode of social cognition.

Of course, mindreading theorists maintain that much of mindreading is not consciously accessible, so an appeal to phenomenology is not so straightforward. Phenomenologists recognize that phenomenology cannot demonstrate directly whether certain sub-personal processes are operative. However, they argue that it can serve as indirect evidence about sub-personal processing. Articulating this view, Shaun Gallagher argues: 
Phenomenology cannot tell us whether our response to the exasperation in a person's voice involves an implicit (sub-conscious) theory or pretend belief. But a careful and methodical phenomenology should be able to tell us whether, when we hear the exasperated voice, our usual response involves formulating an explanation or predicting what the person will do next. Our encounters with others are in fact not normally occasions for theorizing or simulating if such nonconscious procedures are cashed out phenomenologically as explaining or predicting on the basis of postulated mental states (Gallagher, 2001, p. 89). ${ }^{4}$

The claim is that if non-conscious mindreading is going on, it should result in phenomenological experience of third-person-based explanation and prediction. Phenomenology tells us that our normal intersubjective experiences are not best characterized as third-person-based explanation and prediction. Hence, phenomenological considerations indicate that non-conscious mindreading is not operative in our normal intersubjective experiences.

In response to this argument, mindreading proponents question the reliability and relevance of phenomenology in this context, specifically challenging the idea that nonconscious mindreading would manifest in conscious, explicit explanation and prediction (Herschbach, 2008b; Jacob, 2011; Spaulding, 2010). In a previous article, I have articulating these objections:

${ }^{4}$ For a more recent defense of these claims, see Gallagher (2012). 
The debate in mindreading between the [TT] and the [ST] is a debate about the architecture and sub-personal processes responsible for social cognition. Neither account is committed to any view on what phenomenology tells us is going on in our ordinary interactions. With mindreading, there is a process (theorizing or simulating), and there is a product (an explanation or a prediction). In general, neither the process nor the product need be consciously accessible, let alone phenomenologically transparent (Spaulding, 2010, p. 131).

Mindreading proponents hold that the phenomenological fluidity of our ordinary interactions does not undermine the claim that mindreading is an important and pervasive way that we understand others. After all, many cognitive activities are phenomenologically fluid yet realized by complex computational processes that involve inferential processing at the sub-personal level. Language and vision, it is argued, are two clear examples of this (Spaulding, 2010, p. 135).

Phenomenologists offer three related replies to these arguments. First, they argue that it does not follow from the fact that phenomenological experience sometimes is unreliable that it always is. Careful phenomenological analysis may not be subject to these concerns about reliability. Second, generalizing this line of argument results in the unpalatable conclusion that phenomenology never helps us understand how our cognitive mechanisms work and we never should base our arguments on phenomenological considerations (Gallagher, 2012, p. 203). Third, phenomenology in fact is necessary for understanding social cognition. Dan Zahavi, for example, argues that accounts of social cognition are supposed to explain the full diversity of personal-level social cognition. 
If we don't know the explanandum well, if we don't possess careful descriptions of its different facets - which is arguably one of the things that phenomenology can offer - it will, to put it mildly, be kind of hard to assess the relevance and explanatory power of the sub-personal mechanisms (Zahavi, 2011, p. 555).

According to this view, phenomenology helps us understand personal-level social cognition, which is necessary to assess hypotheses about sub-personal cognitive processes. Thus, far from being irrelevant, phenomenology is necessary for the study of social cognition.

From this relatively brief summary, we can see the outlines of the debate between the phenomenologists and mindreading proponents. Mindreading proponents argue that phenomenological evidence is too unreliable to be useful and is irrelevant to the questions at issue. Phenomenologists hold that phenomenology can be reliable and is part of the explanandum of social cognition theories, and this warrants giving phenomenology a significant methodological role.

To resolve this debate, we need to determine whether phenomenology strongly constrains the study of social cognition. For phenomenology to play a substantial methodological role, it ought provide novel, reliable, and relevant information. The next three sections address these criteria. I shall argue that phenomenological evidence lacks all three characteristics, and thus it has no special evidential role in social cognition debates.

\section{The Novelty of Phenomenological Evidence}


Theory choice always is comparative. When testing a theory, we always are testing it against some available alternative theory. The criterion that evidence be novel should be understood in this comparative context. Evidence is novel to the extent that it provides information beyond what is widely acknowledged and presupposed by the theories debated.

The novelty criterion matters for two related reasons. First, if the evidence is not novel, if it is taken into consideration either explicitly or implicitly by all the theories debated, then it would inappropriate to argue that one of the theories debated is not supported by that evidence. That evidence not only is compatible with the theory, it is part of the background information used to construct the theory. In the case at hand, if a theory of social cognition takes for granted that ordinary social interactions have a certain phenomenology, then (so long as we agree on the phenomenological nature of our interactions) the objection that the theory is not supported by our phenomenological experience appears misguided.

The second reason novelty is important involves the distinction between mere accommodation of previously known data and novel prediction (Hitchcock \& Sober, 2004). A theory accommodates some phenomenon if the theory was constructed specifically to predict that phenomenon. A phenomenon is novel to a theory only if the theory was not constructed specifically to predict that phenomenon. Accommodating data is not detrimental for a theory. After all, we do not expect empirical theories to be constructed $a$ priori, and fit with existing data is a good thing. Nevertheless, accurately predicting some phenomenon beyond that which it was constructed to predict is the mark of a good theory. 
There is a long-standing debate in the philosophy of science between predictivists and non-predictivists about whether the prediction of novel phenomena provides better epistemic support for a theory than the accommodation of previously known data. Without wading too deeply into this debate, I suggest that a theory's ability to accommodate previously known data is weak support for that theory. Moreover, when deciding amongst theories, accommodating previously known data is an advantage only if the competitor theories neither accommodate nor predict those data. Thus, accommodating phenomenological evidence is an advantage for phenomenological theories only if mindreading theories neither accommodate nor predict the phenomenological evidence.

Phenomenological evidence is not novel to mindreading theories because they are constructed to be compatible with such evidence. Although mindreading proponents do not provide detailed accounts of our phenomenology of social interactions, and they do not regard phenomenology as a good guide to the nature of our cognitive systems, their descriptions of the theories reflect the presupposition of common phenomenological evidence. For example, in one of the foundational articles on theory of mind, Simon Blackburn explains the relationship between mindreading and phenomenology.

Theory [of mind] plays an explanatory role, not a phenomenological one. It is not that ... the listener finds himself making an inference or indulging a train of reasoning. Obviously no such conscious process occurs: this is what makes the judgement spontaneous. But it may nevertheless be true that there exist principles to take us from evidence to interpretation; that these principles can be cited when dispute or demand for justification arises; that it was necessary to 'internalise' those principles to become 
a trained or well tuned observer; and that one learned the form a 'network of corrigible assumptions', or in short a theory (Blackburn, 1992, p. 192).

Additionally, in a widely cited collection of essays on the TT and the ST, Martin Davies and Tony Stone describe the TT in the following way.

When I predict what someone will do, or explain why they have done something, I do so by deploying this theory. Most of us are, of course, quite unaware that this is what we are doing; but, as with grammatical theory, the fact is reckoned to be unimportant. Our relationship to the psychological theory is allowed to be 'tacit' or 'implicit' knowledge (Davies \& Stone, 1995, p. 2).

Davies and Stone draw an analogy between mindreading and the processing of grammar, both of which involve complex computational processes but no phenomenological experience of these complex computational processes. I highlight these quotes to show that from the early days of the mindreading debate theorists distinguished between cognitive mechanisms and phenomenological experiences. Such remarks are not limited to the TT. Simulation theorists also are careful to point out that many mindreading simulations take place subconsciously (Goldman, 2006, p. 161; Gordon, 1992, p. 12).

One can find many instances of mindreading theorists cautioning that the mechanisms they describe need not and often do not operate consciously and explicitly. Such qualifying remarks indicate that mindreading theories presuppose that our ordinary social interactions often seem to involve no effortful deliberation about mental states. 
These comments reflect awareness of the basic phenomenological character of ordinary social interactions and serve to clarify that the TT and the ST do not mistakenly entail certain phenomenological experiences. If mindreading theorists did not presuppose certain commonplace phenomenological evidence, they would not need to make these clarificatory remarks about our conscious experiences. Thus, phenomenological evidence is widely acknowledged and presupposed by mindreading theories. ${ }^{5}$

As I noted above, phenomenological theories' ability to accommodate our phenomenological experience is an advantage only if the competitor theories neither accommodate nor predict our phenomenological experience. However, as these comments indicate, mindreading theories also accommodate basic phenomenological evidence. They presuppose commonly accepted phenomenological claims and construct the theories to be compatible with this evidence. Consideration of these points indicates that phenomenological evidence is not novel in the relevant sense, and thus it lacks the first feature of good evidence.

Although mindreading proponents presuppose commonly accepted phenomenological claims, phenomenologists and mindreading theorists take very different perspectives on phenomenological evidence. Unlike phenomenologists, mindreading theorists do not take phenomenology to be an important source of evidence about social cognition. Rather, phenomenology is regarded as extremely defeasible for two reasons. First, phenomenological reports about our social interactions vary widely. Some people

${ }^{5}$ Despite these examples, not all mindreading proponents carefully distinguish claims about cognitive mechanisms from claims about our conscious experiences. Phenomenologists' recent critiques of mindreading have been a useful corrective to this sloppiness. Nevertheless, mindreading proponents can, should, and many do distinguish phenomenological claims from claims about cognitive architecture. 
report regularly thinking about others' mental states, others report such thinking only when a social interaction breaks down, and others report that they almost never are consciously aware of thinking about others' mental states. In the next section I will discuss in more detail the variability of phenomenological reports. Mindreading proponents do not put much stock in phenomenological reports because of their variability.

Second, mindreading proponents do not regard phenomenology as a good guide to the nature of the underlying cognitive mechanisms. This is due to a standing skepticism about inferences from phenomenology to cognitive architecture. Just as the phenomenological fluidity of visual and linguistic processing fails to reflect the complex nature of vision and language, phenomenology of social interactions may be equally misleading. The gap between phenomenological experience and the cognitive mechanisms responsible for social cognition impels mindreading proponents to regard phenomenology as at best provisional evidence in social cognition debates.

In sum, mindreading proponents take for granted some basic, common phenomenological experiences, e.g., that we may not always feel like we are engaged in effortful deliberation about theoretical causal states, and sometimes we have the feeling of simply knowing, without deliberation, why a target is behaving in a certain way and what she will do next. This entails that phenomenological evidence is not novel in the relevant sense. However, mindreading proponents regard these and other phenomenological claims as extremely defeasible because of concerns about their reliability and relevance. Thus, the debate about the methodological role of phenomenology hinges on whether phenomenological evidence is reliable and relevant. 


\section{The Reliability of Phenomenology}

The phenomenological evidence at issue consists in first-person reports of one's conscious experience of social interactions. This section considers whether phenomenological evidence is reliable. I evaluate reliability along three dimensions: consistency, accuracy, and objectivity. ${ }^{6}$ Below is a description of these dimensions of reliability.

- Consistency: Phenomenological reports are consistent to the extent that they are similar for similar interactions.

- Accuracy: A subject's reported phenomenology is accurate to the extent that it provides a factually correct description of nature of the experience.

- Objectivity: A subject's phenomenological report is objective to the extent that it is not significantly biased by evidentially irrelevant factors.

The consistency dimension can be understood intrapersonally or interpersonally. A subject's phenomenological reports are intrapersonally consistent insofar as the subject reports more or less the same phenomenology for similar social interactions.

Phenomenological reports are interpersonally consistent insofar as two (or more) subjects report similar phenomenology for similar interactions.

\footnotetext{
${ }^{6}$ Reliability sometimes is conflated with publicity, i.e., intersubjective testability. Evidence from public methods may be validated as reliable more easily than evidence from private methods. Although I regard publicity as important - perhaps critically important - for whether evidence counts as scientific, in this context it would be question begging to require publicity because phenomenology simply is private (Dennett, 2003; Goldman, 1997).
} 
These qualities sometimes cluster together insofar as accurate reports tend to be consistent and unbiased, and inconsistent reports tend to be inaccurate and biased. However, they are distinct qualities and can come apart in various ways. A particular set of reports may be intrapersonally consistent yet interpersonally inconsistent. That is, one person may consistently report a certain kind of experience whereas others report different experiences in similar situations. Alternatively, a set of reports may be intrapersonally and interpersonally consistent but inaccurate. For example, classic experiments on introspection show that in particular circumstances most people confabulate reports of their conscious experience (Nisbett \& Wilson, 1977). In these cases, subjects consistently report a certain kind of experience that we know to be false. A third possibility is that a set of reports is consistent and accurate yet biased. In such a case, due to a selection, reporting, or interpretive bias, the set of reports does not fairly represent the target phenomena.

For phenomenological evidence to play a substantial methodological role, it ought to be reliable. Evidence is reliable to the extent that it is consistent, accurate, and unbiased. Consider consistency first. Presumably similar social cognitive processes are operative in subjects who are in similar social interactions. If reports are intrapersonally and interpersonally consistent, we can extrapolate from these reports to general claims about cognition even if the reports are inaccurate and biased. However, if subjects' reports are intrapersonally or interpersonally inconsistent, then we cannot extrapolate from these reports to conclusions about social cognitive processes in general. This is not to deny that individuals experience the social world differently. Some individuals may engage in 
conscious, deliberative mindreading more or less than others, of course. But without some level of consistency, empirical generalizations are difficult to make.

Inconsistency could be mitigated if we had criteria for judging the accuracy of the reports. That is, so long as we can judge which phenomenological reports are accurate, modest amounts of variability in phenomenological reports are not problematic. With accuracy criteria, we could differentiate inaccurate reports from accurate reports of idiosyncratic social experiences. We do not always have direct, objective measures of accuracy for first-person reports. Sometimes we have indirect accuracy measures, such as testing for behavior that one would expect if the report were true. In other cases, we lack even those indirect measures of accuracy. This situation is not terribly problematic if the reports generally are consistent. However, if reports are inconsistent and we lack even indirect measures of the accuracy of the reports, this is terribly problematic.

Finally, if phenomenological reports are subject to systematic bias, they do not provide good data on the nature of social interactions. Of course, all evidence is subject to interpretive bias because all evidence must be interpreted by theorists. Any piece of evidence may be skewed by a theorist's interpretation of that evidence. The point at issue here is not whether theorists interpreting the reports are biased, but whether the generation of the reports themselves is biased. That sort of bias is acutely problematic because it is much more difficult to eliminate than a theorist's interpretive bias.

Now that we have an account of the dimensions of reliability, we can examine whether phenomenological reports on social interactions are reliable, i.e., whether they are consistent, accurate, and objective. Scientific data on this topic are very scarce. As of now, there is only one published scientific study on the phenomenology of social cognition, and 
it does not address the reliability of phenomenology (Bryant, Coffey, Povinelli, \& Pruett Jr, 2013). Thus, we must look elsewhere for evidence on the reliability of phenomenology of social cognition. The empirical literature on the phenomenology of other sorts of cognition is fairly well developed. Below I shall discuss the reliability of phenomenological reports in general and then turn to phenomenological claims about social cognition specifically.

The general track record for phenomenological reports is fairly poor. An extensive empirical literature details the circumstances under which our phenomenological reports are unreliable. A thorough examination of that literature requires more space than I have here. I defer to Eric Schwitzgebel's (2008) comprehensive review of the empirical study of phenomenology. Schwitzgebel details the various conditions under which phenomenology has been tested and concludes that phenomenology generally is unreliable.7 Schwitzgebel's review is thorough and broadly acceptable, though theorists who are sanguine about the prospects for phenomenology may draw a different conclusion from these data.

Schwitzgebel contends that our phenomenological capacities fail in the following tasks. We fail at assessing the causes of our mental states and the processes underwriting them. Our judgments about non-phenomenal mental states (e.g., traits, motivations, and skills) are inaccurate and biased. Our judgments about our occurrent conscious experiences are unreliable when we are distracted, passionate, inattentive, self-deceived, and pathologically deluded, and when we're reflecting on minor matters, the past, the current moment, and where fine discrimination is required. We are ignorant and prone to error in basic phenomenological tasks, e.g., reporting what we think and want. We

\footnotetext{
${ }^{7}$ Schwitzgebel reviews the literature on introspection, but there is considerable overlap between introspection and phenomenology. He concludes that the method by which we normally reach judgments about our conscious experiences typically is unreliable.
} 
misjudge fundamental and pervasive aspects of our conscious experiences, e.g., in the absence of environmental cues, determining the emotion or sensation we are experiencing. Moreover, there is no evidence of expertise effects, i.e., no evidence that the reliability of our phenomenological reports improves with practice. Even experts on phenomenology profoundly disagree about the nature of phenomenological experience, e.g., whether a thought has a distinctive phenomenology beyond the imagery invoked by thinking the thought (Schwitzgebel, 2008).

Schwitzgebel's review suggests that phenomenology often is inconsistent, inaccurate, and biased. This does not imply that phenomenology always is unreliable for every task and under every condition. It is an open empirical question when, if ever, phenomenology is reliable. The studies Schwitzgebel reviews do not focus on the phenomenology of social cognition. As mentioned above, there is almost no empirical literature on the phenomenology of social cognition. Phenomenologists' claims result from their introspective examinations of their own experiences of social interactions. (Gallagher, 2012; Zahavi, 2011). This is problematic for several reasons.

First, there is no empirical evidence that even expert phenomenologists are reliable about social cognition. When I ask philosophers to introspect on their phenomenological experience of social interactions, they report widely differing phenomenological experiences. Some report that they almost never think about others' mental states, and others report that they frequently think about others' mental states. Presumably similar 
social cognitive processes are operating in these cases, yet phenomenological reports differ drastically. ${ }^{8}$

Second, introspection notoriously is unreliable when one is introspecting past experiences and forming generalizations about one's experiences. And this is exactly what phenomenologists are doing when they generate claims about their phenomenology of social interactions.

Third, responses to phenomenological questions inherently are biased; they always are subject to the reporting bias. As Elizabeth Irvine observes, "First person reports are the products of decision making about whether a particular stimulus strength warrants a particular response in a given context" (Irvine, 2012, p. 640). This feature of first-person reports is particularly acute here because there is no objective measure against which we can compare phenomenological reports of social interactions. Although both behavioral and subjective reports may be subject to bias, with behavioral evidence we have some objective measures against which we can compare the behavioral evidence. Currently we have no objective measures for subjective phenomenological reports. We cannot eliminate or correct for biases, because we have no objective baseline for comparison.

As Schwitzgebel's review shows, phenomenology generally is unreliable. There is no reason to expect phenomenological reports of social interactions to be more accurate than the array of phenomenological reports reviewed. Moreover, given that phenomenological reports of social cognition primarily result from solitary theorists' introspective reports on past experiences, a method with well-documented flaws, it seems that phenomenological

${ }^{8}$ Like mindreading theorists, phenomenologists presuppose that, for most individuals, there are similar social cognitive processes operating. They argue that the phenomenological evidence suggests that the TT and the ST are incorrect theories, not that they simply fail to describe their own phenomenological experience. 
reports of social interactions are just as subject to these reliability problems as other phenomenological reports.

It is worth discussing the results of the one empirical study on the phenomenology of social cognition (Bryant et al., 2013). This study, by Lauren Bryant and colleagues, employs Descriptive Experience Sampling (DES) to investigate how often subjects consciously find themselves thinking about others' mental states. In the study, 30 undergraduate students each wear a beeper for 10 hours. The subjects are instructed to write down whatever they are thinking whenever the beeper goes off. This method allows researchers to randomly query subjects about their thoughts throughout the day. The reported thoughts are sorted into three categories: action-related thoughts, mental staterelated thoughts, and miscellaneous thoughts. The results of the study are as follows: $47 \%$ of the reported thoughts were about actions, $32 \%$ were about mental states, and $21 \%$ were miscellaneous thoughts.

The result that $32 \%$ of subjects' thoughts were about mental states does not sit well with phenomenologists' claim that we rarely engage in mindreading. ${ }^{9}$ Nevertheless, this study has only limited significance for this discussion. First, mindreading proponents argue that much mindreading is subconscious. Even if the reports are completely reliable, they cannot tell us how often we engage in mindreading because they tell us nothing about subconscious processes. Second, the study reveals very little about the reliability of phenomenology. This study does not address the consistency or accuracy of

\footnotetext{
${ }^{9}$ Thinking about mental states is not the same as mindreading, which additionally involves the attribution of mental states. Phenomenologists argue that we are more immediately engaged in social interactions than mindreading theories indicate. We rarely take a thirdperson perspective on mental states, they argue. The fact that, at least in this study, $32 \%$ of subjects' thoughts are about mental states is in tension with this argument.
} 
phenomenological reports. The DES methodology in principle can give us evidence about intrapersonal and interpersonal consistency (though this study does not), however the method cannot validate the accuracy of phenomenological reports. Third, and relatedly, the results are subject to the reporting bias that Irvine discusses. Thus, this study cannot help us resolve our question about the methodological role of phenomenology in social cognition debates.

Nevertheless, additional DES studies on social cognition would be useful. This methodology is an enormous improvement over the current armchair method of generating phenomenological claims about social cognition. DES controls for some of the most problematic elements of the current method, e.g., reflection on past experiences and forming generalizations about one's experiences (Engelbert \& Carruthers, 2011; Hurlburt \& Schwitzgebel, 2007; Weisberg, 2011).10

In light of evidence about the general unreliability of phenomenology, however, we should treat the results of this and future DES studies with caution. We need not assume that the reported thoughts reliably reflect the actual experience. We should treat these phenomenological reports as extremely defeasible reports on what subjects believe themselves to be experiencing.

\footnotetext{
${ }^{10}$ In addition to DES, think-aloud tasks, which require subjects to verbally talk through their experiences, may be a useful addition to the empirical literature on social cognition. One difficulty with directed requests for verbal reports (such as in the standard false-belief task) is that the expectation of delivering information to the experimenter alters the thought process itself, which affects the reliability of the reports. A recent meta-analysis of think-aloud studies suggests that the think-aloud protocol is not subject to this reliabilitycompromising effect (Fox, Ericsson, \& Best, 2011). Like DES, the think-aloud protocol is incapable of directly shedding light on unconscious cognitive processes. Nevertheless, both methodologies would be welcome additions to the social cognition literature.
} 
Treating first-person reports as a defeasible guide to subjects' experiences is not a revolutionary idea. It is, in fact, fairly commonplace, and there are many different ways in which one might develop the idea. For example, Daniel Dennett, Gualtiero Piccinini, and Alva Noë offer distinct accounts of how to take seriously subjects' phenomenological reports without assuming that what subjects report is accurate and unbiased (Dennett, 2003; Noë, 2007; Piccinini, 2009). Whichever account one adopts, this seems like the most sensible option in light of the extensive evidence of the unreliability of phenomenology. A further advantage is that it avoids an objection to the unreliability argument mentioned earlier. Gallagher argues that generalizing the worry about the reliability of phenomenology results in the unpalatable conclusion that phenomenology never helps us understand how our cognitive mechanisms work and we never should base our arguments on phenomenological considerations (Gallagher, 2012, p. 203). Adopting the perspective that phenomenological reports are defeasible guides to subjects' experiences avoids this conclusion. Phenomenological reports may be useful when they are understood appropriately even though they are extremely defeasible.

There are several good reasons to adopt something like Dennett, Piccinini, or Noë's account. Notice, though, to go this route is to give up on the idea that phenomenology plays a substantial methodological role in social cognition debates. Phenomenological reports are, on this view, frequently unreliable reports of what one takes oneself to be experiencing. That is not the sort of evidence that straightforwardly can confirm or disconfirm theories of social cognition.

\section{The Relevance of Phenomenology}


The case for phenomenology hangs on the relevance of phenomenology to social cognition debates. On one hand, if phenomenology turns out to be relevant, we should regard the unreliability of phenomenology simply as a limitation of our current methodologies and continue to develop better methodologies. ${ }^{11}$ Perhaps in the future we will figure out an experimental paradigm that controls for some of the difficulties with consistency, accuracy, and biased nature of phenomenological reports. On the other hand, if phenomenology turns out not to be relevant to social cognition debates, surely that would be a decisive blow to the phenomenological approach. If phenomenological evidence is old, unreliable, and irrelevant, nothing justifies granting it a substantial methodological role in this debate.

Mindreading proponents maintain that phenomenology is irrelevant to social cognition debates insofar as these debates are about the cognitive architecture and subpersonal processes responsible for social cognition, and phenomenology cannot shed light on these issues (Carruthers, 2009; Currie, 2008; Herschbach, 2008b; Jacob, 2011; Spaulding, 2010). Defending the relevance of phenomenology, phenomenologists point out that if it really were totally irrelevant to social cognition, our theorizing never could get off the ground (Gallagher, 2001; Ratcliffe, 2006; Zahavi, 2011, p. 555). In a recent articulation of this view, Gallagher argues, "It is not unusual for neuroscientists to appeal to personal

${ }^{11}$ Phenomenology - first person reports of one's conscious experiences - clearly is relevant for some areas of research. Phenomenology is the primary explanandum of theories of consciousness, and it is essential for investigating psychiatric conditions. For example, depression, schizophrenia, and synesthesia are defined in terms of and clinically identified by specific kinds of phenomenological reports. Theories about consciousness, depression, schizophrenia, and synesthesia make predictions about occurrent conscious experiences, and thus phenomenology is a crucial aspect of theorizing about these conditions. Theorists in these research areas must contend with the concerns about the reliability of first-person reports and work to find improved methodologies. 
level practices and phenomenological experiences in setting up their experiments, and in many cases the only way to define the explanandum is in terms of phenomenology. For example, if a neuroscientist wants to study brain processes that correlate with empathy she needs to have something better than a wild guess about what the experience of empathy is and what behavioral situations might elicit it" (Gallagher, 2012, pp. 205-206). The claim is that without phenomenology, we could not even begin to explain social cognition or assess the relevance of sub-personal cognitive processes to social cognition.

The idea that we must have some understanding of the phenomenology of social interactions in order to theorize about social cognition is true, but it sidesteps the real dispute. As I argued in section 3, some basic phenomenological claims are and always have been implicit in the construction of mindreading theories. Mindreading theorists need not deny that we need some understanding of a typical experience of a social interaction in order to identify the explanandum. The real point of disagreement is whether phenomenology is relevant in a more substantial sense. Can phenomenology confirm or disconfirm hypotheses about social cognition? Put another way, do theories of social cognition make claims that are testable phenomenologically?

Phenomenologists answer affirmatively. Gallagher states, "If I am constantly predicting and explaining the behavior of others, it would seem odd to claim that I'm not sometimes aware of it, especially, for example, when my predictions or explanations fail, that is, when I fail to understand the other person, which is not an infrequent event" (Gallagher, 2012, p. 207). In a similar vein, Matthew Ratcliffe claims, "It could be added that, if theory and simulation are primarily about enabling prediction and explanation, the phenomenological absence of prediction and explanation also constitutes a case against the 
sub-personal operation of [folk psychology] in the form of theory or simulation" (Ratcliffe, 2006, p. 36).

The claim is that if we really were explaining and predicting behavior all the time as mindreading proponents argue, we should have some phenomenological experience of this. But, phenomenologists argue, a careful phenomenological analysis reveals that we are not constantly explaining and predicting behavior. According to this argument, phenomenology is relevant because mindreading theories predict phenomenological experiences (namely, explaining and predicting) that we do not have.

The fundamental issue here is whether explanation and prediction exclusively are personal-level processes of which we always are consciously aware. Gallagher explicitly maintains that they are. "Explanation (or theory) seems to mean (even in our everyday psychology) a process that involves reflective consciousness. The term 'prediction' also seems to me to describe a reflective conscious act... 'Explanation' and 'prediction' are personal-level terms" (Gallagher, 2005, p. 215). Phenomenologists maintain that mindreading theories entail that we often have certain kinds of conscious experiences, namely, that of explaining and predicting behavior. Phenomenology shows, however, that we rarely have such conscious experiences.

Mindreading proponents, in contrast, use the terms "explanation" and "prediction" in a broader way. In mindreading theories, explanation and prediction also to refer to subpersonal processes that are not consciously accessible (Herschbach, 2008a; Jacob, 2011; Spaulding, 2010). According to this view, many of our social interactions consist in implicit mindreading, i.e., subconsciously explaining and predicting targets' behavior on the basis of attributed mental states. 
Phenomenologists are right to point out the peculiarity of subconscious explanation and prediction. This is, at least in part, a terminological issue. The terms "explanation" and "prediction" commonly are used to characterize mindreading, but these terms are problematic. They are carryovers from conceptualizing social cognition in terms of a literal theory of mind.

Decades ago, philosophers and psychologists studying social cognition argued that we understand others by employing a literal theory of mind, which involves folk psychological laws that connect mental states, unobservable theoretical entities, to behavior. Much of social cognition, it was argued, consists in employing these folk psychological laws, along with auxiliary assumptions about the relevant circumstances, to deduce explanations and predictions of behavior. They argued that we use our theory of mind just like, for example, physicists use the theory of gravity to explain and predict the behavior of physical objects. Jerry Fodor, for example, argues that theory of mind explanations "are frequently seen to exhibit the 'deductive structure' that is so characteristic of explanation in real science. There are two parts to this: the theory's underlying generalizations are defined over unobservables, and they lead to its predictions by iterating and interacting rather than by being directly instantiated" (Fodor, 1987, p. 7).

The conception of theory of mind described above has fallen out of favor, as has the Deductive-Nomological model of scientific theorizing on which it is based. ${ }^{12}$ Philosophers of science have abandoned the idea that all, or even most scientific theories consist in

12 Conceiving of theory of mind in terms of the Deductive-Nomological model is specific to traditional formulations of the TT, but generally conceiving of theory of mind in terms of scientific theorizing is neutral between the TT and the ST. Both the TT and the ST employ a kind of theorizing: a systematic process that results in explanations and predictions of some target phenomenon. 
collections of laws from which we deduce explanations and predictions. Similarly, most theorists studying social cognition, even theory theorists, have given up on the idea that social cognition literally involves applying folk psychological laws and deriving explanations and predictions from these laws. Contemporary theory theorists characterize our social cognition as an information rich, interpretive process, which stands in contrast to simulation theory's information poor, simulational process. Thus, the Deductive Nomological model of theory of mind is outdated. Even the term "theory of mind" is becoming outdated. Because of its theoretical connotations, some theorists advocate using the term "mindreading" instead.

The contours of the mindreading debate have shifted over the decades, and this is reflected in the changing terminology. "Explanation" and "prediction" are terminological hangovers from the Deductive-Nomological model of mindreading, and as such they should be retired as well. To avoid these terminological disputes about subconscious explanation and prediction, we need more appropriate terms to describe the cognitive processes involved in mindreading. Below I propose "interpretation" and "anticipation" as more appropriate and neutral terms for mindreading. These suggestions may or may not turn out to be the best alternatives, but it is past time to come up with new terminology for explanation and prediction. ${ }^{13}$

A more fitting alternative to the term "explanation" is "interpretation." Mindreading is interpretation that involves attributing mental states. Interpretation, as I use the term, is

\footnotetext{
${ }^{13}$ In a similar vein, some theorists have urged a terminological shift to "online" and "offline" social cognition. See, e.g., Herschbach (2008a), Dumontheil, Apperly, and Blakemore (2010), and Schilbach (2014). The online/offline distinction does not map exactly onto the distinction I make, though I think reconceptualizing social cognition in these terms is move in the right direction.
} 
the process of taking in information and selectively attuning to certain aspects of that information in order to model the target phenomenon. The kind of model involved here is abstract; it is not a physical entity or linguistic description. It may be a heuristic, stereotype, or prototype. Interpretation is vulnerable to error in two ways. The informational aspects to which one is attuned may not accurately represent the phenomena, and the imposed model may be a poor fit. There may not be a single best model for a particular phenomenon; nevertheless some models may be more appropriate given the pragmatic context.

One can model a target phenomenon by way of simulation, which proceeds roughly as stipulated by the ST of mindreading. One observes a target's behavior, attunes to certain aspects of that behavior, and mentally simulates engaging in that behavior. The result is an attribution of mental states that could have caused the behavior. Simulational modeling is one way to interpret a target phenomenon. Alternatively, one can model a target phenomenon by way of a theoretical process. The theoretical process could be spelled out in numerous ways, as various models of theorizing have emerged since the demise of the Deductive Nomological model. Below I describe one promising prospect for theoretical modeling.

Heidi Maibom $(2007,2009)$ distinguishes three types of models that are useful for understanding social interpretation as theoretical modeling: models for goal-directed behavior, social models, and folk psychological models. Theoretical models specify a general structure, relations, and properties of some phenomenon. Consider, for example, the social model for a university. The university model represents the social structure of a university in terms of its overall purpose (providing postgraduate education to adults) and 
circumscribes individuals' roles within the university (professors, students, administrators). Facility with the university model helps one understand behavior of a university and the individuals in the university, even if one knows little about the particular institution to which the model is applied. On this view, interpretation consists in constructing and applying a given model on the basis of similarities between the informational aspects to which one is attuned and that model.

With respect to mindreading, one constructs and applies what Maibom calls folk psychological models. There is a single, core folk psychological model, which consists in the distinction between beliefs and desires, the idea of sensory input and behavioral output, and the dependence of action on perceptions, memories, goals, etc. The core folk psychological model can be elaborated, updated, and altered in various ways, and we can build model psychological profiles for particular social roles, groups of people, or individuals. On this account of interpretation, theoretical modeling involves attuning to certain aspects of a phenomenon (what the target is saying and doing, her recent history, personality, etc.) and applying to the phenomenon a folk psychological model that resembles the target phenomenon.

For both simulational modeling and theoretical modeling, it takes practice to learn how to apply to the models, i.e., to recognize patterns in behavior and apply appropriate models to the phenomena. Interpretation ranges from reflectively conscious processes to spontaneous non-conscious processes. In some cases, the model may be explicit and deployed deliberately, as in the case of scientific interpretation, but it need not be. The model may be implicit, and the agent using it may not be able to describe it in any great detail, and thus may not be able to articulate the similarities between the model and the 
target phenomenon. In such a case, the result of the interpretation is that the agent simply sees the target phenomenon as an instance of a more familiar model. Finally, mindreading interpretation involves modeling behavior in terms of mental states. These may be propositional attitudes, such as beliefs and desires, or non-propositional attitudes, such as loving, fearing, hating, and other intentional states, such as seeing and hearing. ${ }^{14}$

So much for interpretation. In place of "prediction" I suggest we use the term "anticipation." For the version of TT described above, anticipation is based on theoretical modeling. A particular folk psychological model will involve certain behavioral expectations. For example, applying the model irate customer to a fellow patron allows one to anticipate certain kinds of behavior in a target, e.g., raising his voice, demanding to speak to the manager, etc. Anticipation for ST is based on the application of the simulational model. I mentally simulate certain aspects of the target behavior, as a result of which I attribute mental states to the target. I mentally simulate what I would do if I had those mental states, and on that basis anticipate that the target will do as I would in my simulational model.

Anticipation does not have the infelicitous connotation of being an exclusively conscious, deliberative process, like prediction does. One can subconsciously anticipate or consciously, deliberately anticipate. Anticipation better reflects the interactive nature of social cognition, than prediction. Prediction connotes a removed, scientific perspective, whereas anticipation has no such connotation. Anticipation more accurately describes some of the newer empirical evidence for mindreading. For example, many developmental

${ }^{14}$ Gallagher (2007) argues, in the context of critiquing the ST, that using a model is an exclusively personal-level phenomenon. As my comments in the main text indicate, I think this is a mistake. See Herschbach (2008b, pp. 228-232) for further defense of sub-personal modeling. 
psychology studies on social cognition involve anticipatory looking, violation of expectation, and active helping conditions. ${ }^{15}$ These tests measure whether young children spontaneously help a target with an uncompleted task, where infants look when observing a target's goal-directed behavior, and how long they look at novel, unexpected visual stimuli. It is more natural to describe these experimental paradigms as testing infants' and children's ability to anticipate a target's behavior.

"Anticipation" and "interpretation" are improvements over "prediction" and "explanation" in three ways. First, anticipation and interpretation are neutral with respect to the TT and the ST. Whereas theory of mind talk, including the associated explanation and prediction terminology, has distinct TT connotations, these terms are neutral.

Interpretation may involve simulational modeling or theoretical modeling, and anticipation does not presuppose one view or the other.

Second, adopting this terminology preempts a misguided objection to mindreading. Some critics argue that mindreading theories regard subjects as mere spectators in social interactions, but phenomenological analysis suggests that we are participants in rather than spectators of social interactions (Hutto, 2008, pp. 1-22). Unlike explanation and prediction, interpretation and anticipation do not give the impression that agents are spectators rather than interacting agents. Players on a football field, for example, are paradigmatic interacting agents, not mere spectators, and it seems perfectly natural to describe the players as interpreting their opponents' movements and anticipating what they will do next.

${ }^{15}$ See Thompson (2012) for a review of this literature. 
Third, and most pertinent to the debate here, "anticipation" and "interpretation" do not automatically render subconscious mindreading conceptually suspicious. Recall that phenomenologists argue that explanation and prediction by definition involve reflective consciousness (Gallagher, 2005; Ratcliffe, 2006; Zahavi, 2011). Whether or not that is correct, it is not part of the concepts of interpretation and anticipation that they involve conscious deliberation. Interpretation and anticipation may be subconscious processes. Moreover, they may involve propositional attitudes, non-propositional attitudes, or other intentional mental states, which mitigates the concern that mindreading is too focused on propositional attitudes like belief and desire. Interpretation and anticipation plausibly allow flexibility in the process and content of mindreading, whereas explanation and prediction do not. In sum, the interpretation and anticipation terminology better reflects both explicit and implicit mindreading and should be adopted as replacements for "explanation" and "prediction."

Recall that phenomenology-based criticisms of mindreading are prefaced on the idea that explanation and prediction exclusively are conscious, personal-level psychological processes, and phenomenology shows that we rarely explain and predict behavior (Fuchs, 2012; Gallagher, 2012; Ratcliffe, 2006; Zahavi, 2011). If, as I have argued, interpretation and anticipation are suitable substitutes for explanation and prediction, and interpretation and anticipation can be subconscious, then the phenomenological objection loses its force. Phenomenology may reveal whether conscious interpretation and anticipation are occurring, but it cannot tell us whether subconscious interpretation and anticipation are occurring. Given that mindreading theorists hold that much of social cognition consists in subconscious interpretation and anticipation, the relevance of phenomenology is limited. 
Mindreading theories generally do not make claims that are testable phenomenologically, however there is one specific area of social cognition that is an exception. Claims about empathy involve phenomenological commitments. There is much debate over what empathy is, the extent to which it is cognitively mediated, its epistemic role in understanding other minds, and how it is realized in the brain. The aspect of empathy that concerns us here is the similarity between a target's and a subject's affective state. If A empathizes with B, it follows that A and B consciously experience a particular affect. Thus, claims about empathy are testable, at least in part, by phenomenology. To take simplistic example, if phenomenological analysis reveals that I am in affective state $\mathrm{X}$ but you in fact are in dissimilar affective state $Y$, then it follows that I am not empathizing with you.

Empathy is not an objection to the thesis advanced in this paper. The study of empathy as a social cognitive capacity need not be focused primarily on phenomenological aspects. It may instead focus on the isomorphism between a subject and a target. At most, empathy is an example of how phenomenology weakly constrains theories about social cognition. This requires that phenomenologically testable claims about empathy are not false. Social cognition theories aim to describe empathy and related concepts, such as sympathy and emotional contagion, and this necessarily involves describing some aspects of our phenomenology. Theories satisfy the weak constraint so long as they do not involve false predictions about our phenomenology. This does not show that theories of social cognition generally ought to explain and be empirically supported by phenomenological evidence. 


\section{Conclusion}

I have argued that in order for phenomenology to play a substantial methodological role in debates about social cognition, phenomenological evidence ought to be novel, reliable, and relevant. However, phenomenological evidence lacks all three characteristics and therefore cannot play a substantial methodological role. This conclusion does not settle the debate between mindreading theories and interactive, embodied, and enactive accounts of social cognition, of course. But it does put to rest a wrongheaded argumentative strategy.

The claim that phenomenology ought not play a substantial methodological role is compatible with wanting our theories of social cognition to provide more rigorous characterizations of the phenomenology of social interactions. It would not be a fatal flaw in these theories if they lacked a detailed account of our phenomenology, but one might want more from our theories insofar as they are meant to describe our experiences of the social world. There is something it is like for me to engage with another person that is different experientially from what it is like for me to engage with inanimate objects. It is compatible with my arguments in this paper to expect a completed theory of social cognition to explain how the sub-personal mechanisms posited enable the distinctive conscious social experiences we have. ${ }^{16}$

\footnotetext{
${ }^{16}$ I am grateful to Mitchell Herschbach, Michael Roche, Robert Thompson and Michael Wilby for their insightful, constructive comments on previous drafts of this paper. Thanks also to the participants at the TESIS Instituting Minds conference in London 2014, where I presented this paper. Finally, thanks to the anonymous referee at this journal. This paper benefited greatly from all the feedback I received from these people.
} 


\section{References:}

Blackburn, S. (1992). Theory, observation and drama. Mind \& Language, 7(1 - 2), 187-230.

Bryant, L., Coffey, A., Povinelli, D. J., \& Pruett Jr, J. R. (2013). Theory of Mind experience sampling in typical adults. Consciousness and Cognition, 22(3), 697-707.

Carruthers, P. (2009). How we know our own minds: The relationship between mindreading and metacognition. Behavioral and Brain Sciences, 32(2), 1-18.

Currie, G. (2008). Some ways to understand people. Philosophical Explorations, 11(3), 211-218.

Davies, M., \& Stone, T. (1995). Folk Psychology: The Theory of Mind Debate. Oxford: Blackwell.

De Jaegher, H., \& Di Paolo, E. (2007). Participatory sense-making. Phenomenology and the Cognitive Sciences, 6(4), 485-507.

Dennett, D. (2003). Who's On First? Heterophenomenology Explained. Journal of Consciousness Studies, 10(9-10), 19-30.

Dumontheil, I., Apperly, I. A., \& Blakemore, S. J. (2010). Online usage of theory of mind continues to develop in late adolescence. Developmental Science, 13(2), 331-338.

Engelbert, M., \& Carruthers, P. (2011). Descriptive Experience Sampling: What is it good for? Journal of Consciousness Studies, 18(1), 130-149.

Fodor, J. A. (1987). Psychosemantics. Cambridge, MA: MIT Press.

Fox, M. C., Ericsson, K. A., \& Best, R. (2011). Do procedures for verbal reporting of thinking have to be reactive? A meta-analysis and recommendations for best reporting methods. Psychological Bulletin, 137(2), 316.

Fuchs, T. (2012). The phenomenology and development of social perspectives. Phenomenology and the Cognitive Sciences, 1-29.

Gallagher, S. (2001). The practice of mind. Theory, simulation or primary interaction? Journal of Consciousness Studies, 8(5-7), 83-108.

Gallagher, S. (2005). How the Body Shapes the Mind: Oxford University Press, USA.

Gallagher, S. (2007). Simulation trouble. Social Neuroscience, 2(3-4), 353-365.

Gallagher, S. (2012). In defense of phenomenological approaches to social cognition: Interacting with the critics. Review of Philosophy and Psychology, 3(2), 187-212.

Goldman, A. I. (1997). Science, publicity, and consciousness. Philosophy of Science, 525-545.

Goldman, A. I. (2006). Simulating Minds: The Philosophy, Psychology, and Neuroscience of Mindreading (Philosophy of Mind): Oxford University Press, USA.

Gordon, R. M. (1992). The Simulation Theory: Objections and Misconceptions. Mind \& Language, 7(1-2), 11-34.

Herschbach, M. (2008a). False-belief understanding and the phenomenological critics of folk psychology. Journal of Consciousness Studies, 15, 33-56.

Herschbach, M. (2008b). Folk psychological and phenomenological accounts of social perception. Philosophical Explorations, 11(3), 223-235.

Hitchcock, C., \& Sober, E. (2004). Prediction versus accommodation and the risk of overfitting. The British Journal for the Philosophy of Science, 55(1), 1-34.

Hurlburt, R. T., \& Schwitzgebel, E. (2007). Describing Inner Experience?: Proponent Meets Skeptic: MIT Press.

Hutto, D. D. (2008). Folk Psychological Narratives: The Sociocultural Basis of Understanding Reasons. Cambridge, MA: MIT Press. 
Jacob, P. (2011). The direct-perception model of empathy: A critique. Review of Philosophy and Psychology, 2(3), 519-540.

Maibom, H. (2007). Social systems. Philosophical Psychology, $20(5), 557$.

Maibom, H. (2009). In Defence of (Model) Theory Theory. Journal of Consciousness Studies, 16, 6(8), 360-378.

Nisbett, R., \& Wilson, T. (1977). Telling more than we can know: Verbal reports on mental processes. Psychological Review, 84(3), 231-259.

Noë, A. (2007). The critique of pure phenomenology. Phenomenology and the Cognitive Sciences, 6(1-2), 231-245.

Piccinini, G. (2009). First-person data, publicity, and self-measurement. Philosophers' Imprint, 9(9), 1-16.

Ratcliffe, M. (2006). 'Folk psychology' is not folk psychology. Phenomenology and the Cognitive Sciences, 5(1), 31-52.

Schilbach, L. (2014). On the relationship of online and offline social cognition. Frontiers in Human Neuroscience, 8.

Schwitzgebel, E. (2008). The unreliability of naive introspection. Philosophical Review, 117(2), 245-273.

Spaulding, S. (2010). Embodied cognition and mindreading. Mind \& Language, 25(1), 119-140. Spaulding, S. (forthcoming). On direct social perception. Pacific Philosophical Quarterly, 1-32. Thompson, J. (2012). Implicit mindreading and embodied cognition. Phenomenology and the Cognitive Sciences, 11(4), 449-466.

Weisberg, J. (2011). Introduction. Journal of Consciousness Studies, 18(1), 7-20.

Zahavi, D. (2011). Empathy and direct social perception: A phenomenological proposal. Review of Philosophy and Psychology, 2(3), 541-558. 